\title{
Bilateral Medial Tibial Plateau Fracture after Arthroscopic Anterior Cruciate Ligament Reconstruction
}

\author{
Ki Cheor Bae, MD, Chul Hyun Cho, MD, Kyung Jae Lee, MD, and Jong Hyuk Jeon, MD \\ Department of Orthopedic Surgery, Keimyung University Dongsan Medical Center, Daegu, Korea
}

\begin{abstract}
Tibial plateau fractures after arthroscopic anterior cruciate ligament (ACL) reconstruction are rare, and only isolated cases have been reported. The authors describe a case of bilateral medial tibial plateau fracture following a minor motorcycle accident in a patient who underwent arthroscopic ACL reconstruction in the past. Two years and four months before the accident, the patient underwent an arthroscopically assisted ACL reconstruction using double-bundle technique on his left knee at a hospital. He had the same surgery using single-bundle technique on his right knee about eight months ago at another hospital. The fractures in his both involved knees occurred through the tibial tunnel and required open reduction with internal fixation. At three weeks after fixation, a second-look arthroscopy revealed intact ACLs in both knees. At five months follow-up, he was able to walk without instability on physical examination. Follow-up radiographs of the patient showed callus formations with healed fractures.
\end{abstract}

Keywords: Tibial plateau fracture, Anterior cruciate ligament, Arthroscopic reconstruction

Anterior cruciate ligament (ACL) reconstruction is a commonly performed procedure that allows recovery of knee stability. Post-surgical complications of ACL reconstruction include stiffness, wound complications, infection, graft failure, deep venous thrombosis, osteonecrosis and peri-articular fractures ${ }^{1,2)}$. However, peri-articular fractures involving the tibial plateau fractures are extremely rare and have been reported only in a few cases ${ }^{3-7)}$. We report an interesting case of bilateral fracture of the medial tibial plateau following a motorcycle accident in a patient who had arthroscopic ACL reconstruction in the past.

\section{Case Report}

A 54-year-old male presented with painful, swollen bilateral

Received November 24, 2014; Revised (1st) February 22, 2015;

(2nd) April 27, 2015; Accepted May 4, 2015

Correspondence to: Ki Cheor Bae, MD

Department of Orthopedic Surgery, Keimyung University Dongsan

Medical Center, 56 Dalseong-ro, Jung-gu, Daegu 700-712, Korea

Tel: +82-53-250-7729, Fax: +82-53-250-7205

E-mail: bkc@dsmc.or.kr

This is an Open Access article distributed under the terms of the Creative Commons Attribution Non-Commercial License (http://creativecommons.org/licenses/by-nc/4.0/) which permits unrestricted non-commercial use, distribution, and reproduction in any medium, provided the original work is properly cited. knees following a minor motorcycle accident where he fell from a motorcycle traveling at a relatively low speed. The accident occurred when a driver backed into the street in front of him riding a motorcycle in an alleyway. He, reportedly, swerved to avoid it and fell from the motorcycle. Radiographs revealed Schatzker type IV tibial plateau fractures involving medial condyles in both knees (Fig. 1). The bone quality was good, and there was no osteopenia. Two years and four months before the accident, the patient had undergone an arthroscopic ACL reconstruction using double-bundle technique with tibialis posterior allograft and medial collateral ligament (MCL) repair on his left knee at a hospital. In spite of our efforts to obtain more information, such as the type of the screw used and tunnel size created in the previous operation, additional information from that hospital was not available. He had the same surgery on his right knee about eight months ago at another hospital. An arthroscopic ACL reconstruction was performed using single-bundle technique with a $25 \mathrm{~mm}$ femur Endobutton (Smith \& Nephew Endoscopy, Andover, MA, USA), a $9 \mathrm{~mm}$ in length and $25 \mathrm{~mm}$ in diameter bio-absorbable interference screw (ConMed Linvatec, Largo, FL, USA), and tibialis posterior allograft. MCL femoral tensioning using an $11 \mathrm{~mm}$ bone staple was performed during the operation with removal of a spiked washer and a screw on the left tibia that had been implanted in the previous ACL reconstruction. Physical 

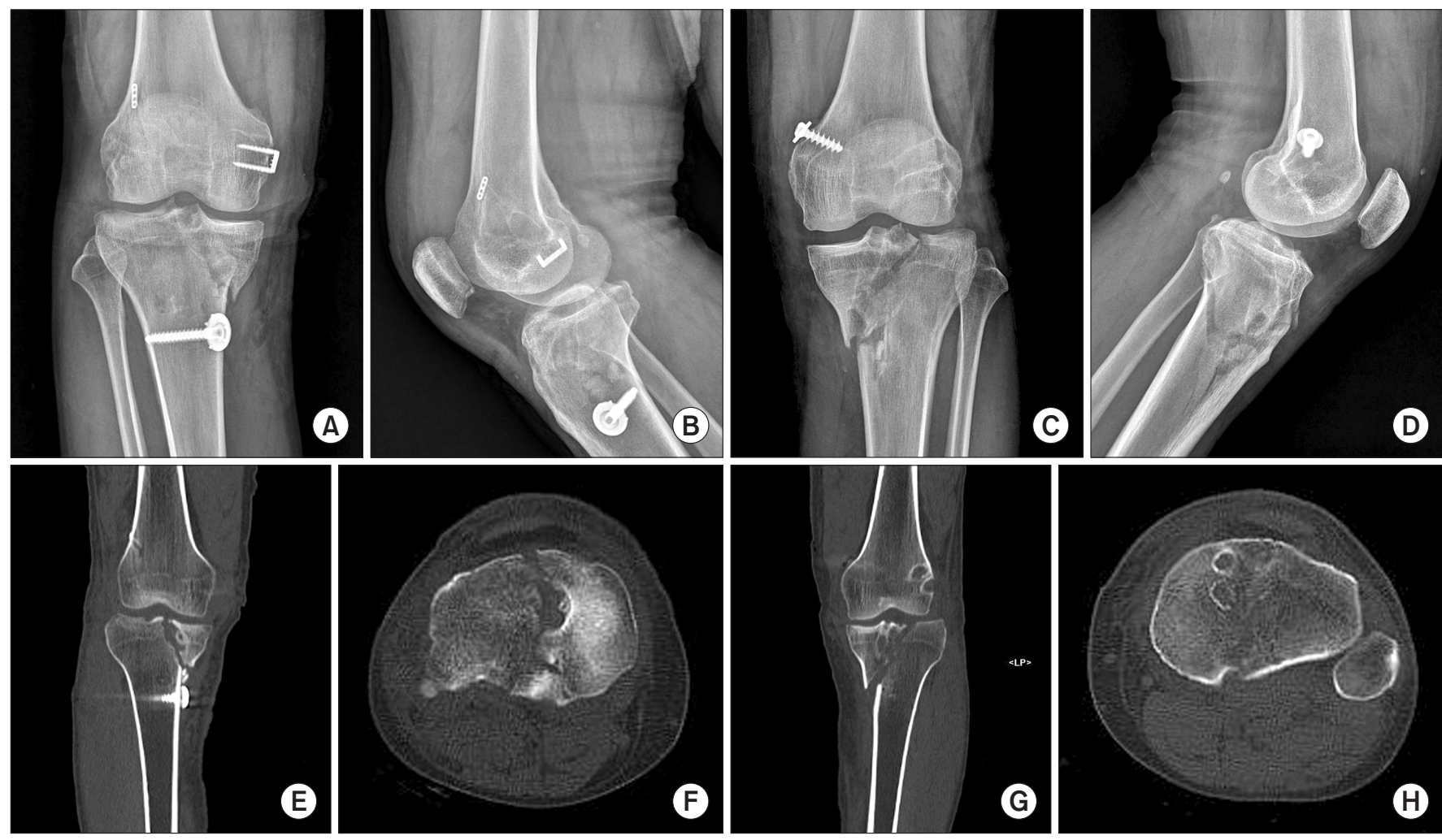

Fig. 1. Preoperative anteroposterior (A) and lateral (B) radiographs of the right knee and anteroposterior (C) and lateral (D) radiographs of the left knee showing tibial plateau fractures involving medial condyles. Coronal (E) and axial (F) computed tomography (CT) scans of the right knee and coronal $(\mathrm{G})$ and axial $(\mathrm{H}) \mathrm{CT}$ scans of the left knee showing evidence of intra-articular tibial plateau fractures involving medial condyles. There are thin fracture lines extending to tibial tunnels.

examination of his both knees showed joint effusion and tenderness with multiple abrasions. Due to severe pain, knee joint stability and range of motion could not be assessed. Computed tomography was performed to evaluate the fracture pattern in relation to the ACL tibial tunnel, which demonstrated intraarticular involvement of split fractures on the bilateral medial tibial plateaus. The fracture lines on his bilateral tibias extended into the tibial tunnels (Fig. 1).

Considering the unique nature of the fractures, we decided to attempt treatment and confirm the state of the previously reconstructed ACL later by second-look arthroscopy. Fracture reduction on the left side was achieved intraoperatively under fluoroscopic control, which was followed by fixation using a six-hole medial locking compression plate (LCP Medial Proximal Tibial Plate; DePuy Synthes, Oberdorf, Switzerland) with a 6.5 cancellous screw and a washer (DePuy Synthes) for anatomical reduction of the intra-articular surface. Subsequently, removal of the spiked washer and screw on the right tibia was done. In the same way, utilizing a four-hole medial locking compression plate (LCP
Medial Proximal Tibial Plate) with a 6.5 cancellous screw and a washer (DePuy Synthes), fracture reduction and fixation on the right side was performed. The planned second-look arthroscopic examination for confirming the previously reconstructed ACL state was achieved three weeks afterwards because the patient had suffered from fever and urinary tract infection since immediately after surgery. Examination under general anesthesia revealed negative anterior Lachmann test and pivot shift test with feeling of firm as the endpoint. The second-look arthroscopic examination revealed intact ACLs on both knees (Fig. 2). However, we could not identify the degree of screw resorption or tunnel widening. Physical therapy with continuous passive motion was started immediately postoperatively, and protected weight bearing was maintained for two postoperative months. At five months follow-up, the patient recovered well and was able to walk. Physical examination revealed no instabilities on both knees. Followup radiographs of the patient showed callus formations with healed fractures in both tibias (Fig. 3). 

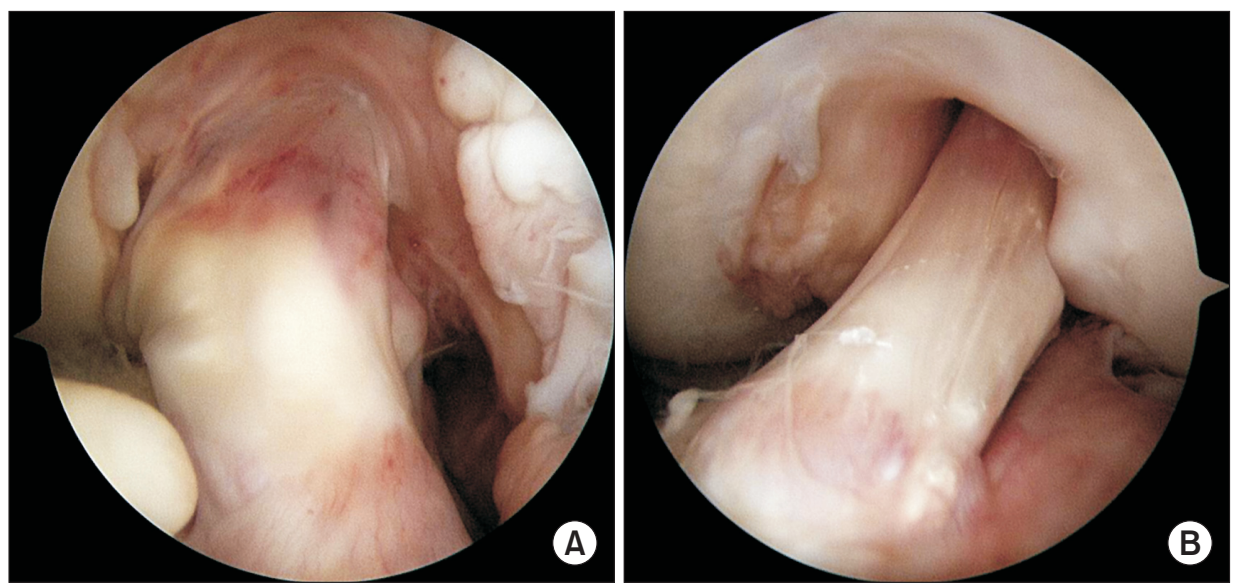

Fig. 2. Second-look arthroscopy of the right knee (A) and left knee (B) showing intact anterior cruciate ligaments.
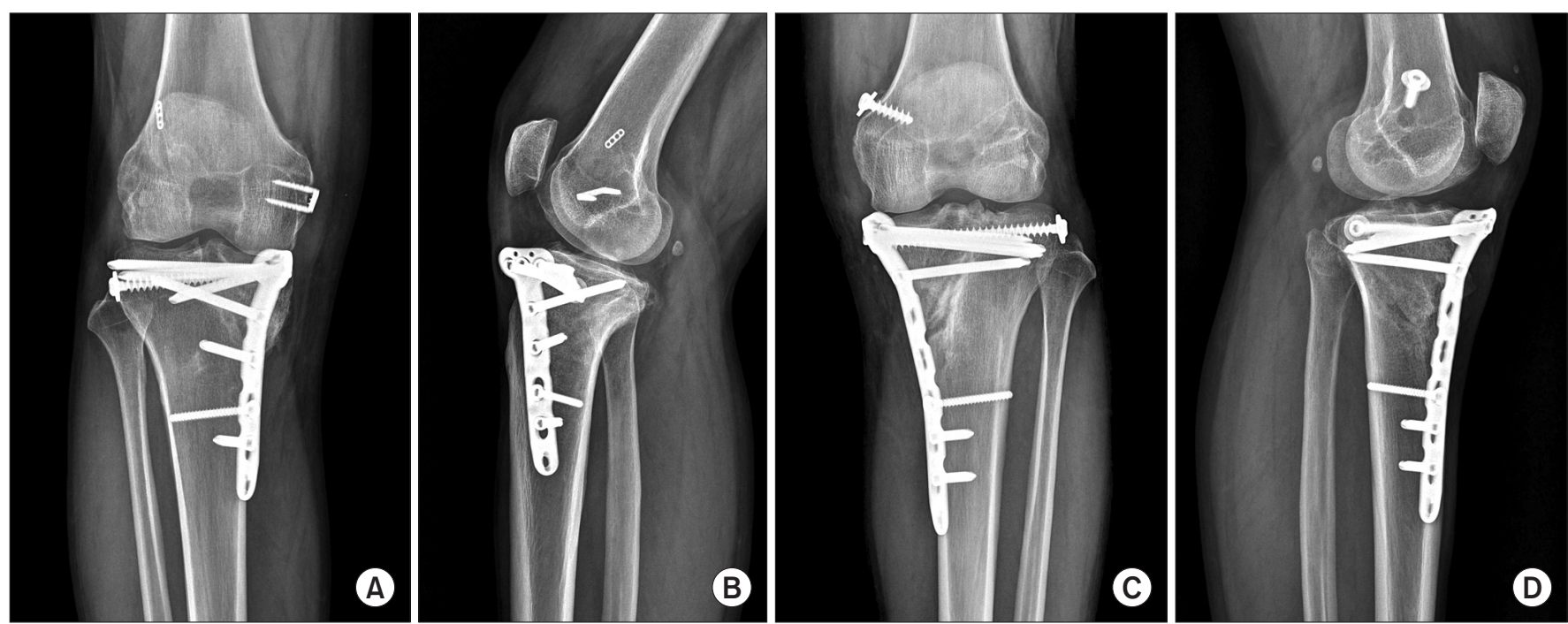

Fig. 3. Anteroposterior (A) and lateral (B) radiographs of the right knee and anteroposterior (C) and lateral (D) radiographs of the left knee showing healed fractures at 5 months follow-up.

\section{Discussion}

Tibial plateau fractures presenting as a serious complication after ACL reconstruction are rare, and only isolated cases have been reported. Interestingly, in most of these cases, the tibial plateau fractures occurred through the tibial tunnel. Authors of these reports suggested that the presence of the tibial tunnel could be the main factor in predisposing the patient to the fracture acting as a stress riser ${ }^{3-7}$. More recently, a case described by A. Gobbi et al. $\left.{ }^{6}\right)$ had some interesting features. Tibial plateau fractures had occurred after primary anatomic double-bundle ACL reconstruction following minimal trauma of running backwards during a soccer game in their case. They suggested that two tunnels made in the proximal tibia might have caused a greater increase in local stresses around the tunnels with less bone available to withstand the applied load compared with a single tunnel. We could not find any studies that specifically measured bone strength of the tibia following tibial tunnel drilling or compared the mechanical effect of two tunnel creation to one tunnel creation. However, it has been documented that bone defects, such as screw holes, can concentrate stress and significantly decrease bone resistance to bending and torsional forces ${ }^{8-9)}$. For instance, diaphyseal drill holes with a diameter greater than $20 \%$ of the bone have been shown to reduce bone strength by $55 \%-90 \%{ }^{8-10)}$.

In our case, the fracture pattern had some unique and distinct features. Although the patient fell from a motorcycle while riding it at a relative low speed, the fractures involved bilateral tibias and occurred through tibial tunnels that had been formed during previous ACL reconstruction. Furthermore, we could not identify other injuries to the articular structures in our patient. He had 
undergone an arthroscopic ACL reconstruction using doublebundle technique on his left knee and the same surgery using single-bundle technique on his right knee. We performed open reduction and internal fixation for the fractures. Fortunately, the ACLs on both knees were found to be intact when examined by second-look arthroscopy. Thus, revision of the ACL reconstruction was not necessary.

Although we were not able to find differences between the effect of single and double-bundle reconstructions on this unique fracture in our patient, it was obvious that the fracture lines on the patient's tibias extended into the tibial tunnel. Our study suggests that a transosseous tibial tunnel alone can somehow act as a stress-riser that decreases strength at the level of proximal tibial metaphysis. We would like to emphasize that the surgeon must be very careful in tunnel placement and should not perform extensive bone removal during ACL reconstruction. Also, it is crucial to be aware of the possible occurrence of this, albeit rare, postoperative complication considering most cases required surgical fixation of the fractures.

\section{Conflict of Interest}

No potential conflict of interest relevant to this article was reported.

\section{References}

1. Allum R. Complications of arthroscopic reconstruction of the anterior cruciate ligament. J Bone Joint Surg Br. 2003;85: 12-6.
2. Jameson SS, Dowen D, James P, Serrano-Pedraza I, Reed $\mathrm{MR}$, Deehan D. Complications following anterior cruciate ligament reconstruction in the English NHS. Knee. 2012;19: 14-9.

3. Delcogliano A, Chiossi S, Caporaso A, Franzese S, Menghi A. Tibial plateau fracture after arthroscopic anterior cruciate ligament reconstruction. Arthroscopy. 2001;17:E16.

4. el-Hage ZM, Mohammed A, Griffiths D, Richardson JB. Tibial plateau fracture following allograft anterior cruciate ligament (ACL) reconstruction. Injury. 1998;29:73-4.

5. Sundaram RO, Cohen D, Barton-Hanson N. Tibial plateau fracture following gracilis-semitendinosus anterior cruciate ligament reconstruction: the tibial tunnel stress-riser. Knee. 2006;13:238-40.

6. Gobbi A, Mahajan V, Karnatzikos G. Tibial plateau fracture after primary anatomic double-bundle anterior cruciate ligament reconstruction: a case report. Arthroscopy. 2011;27: 735-40.

7. Thaunat M, Nourissat G, Gaudin P, Beaufils P. Tibial plateau fracture after anterior cruciate ligament reconstruction: role of the interference screw resorption in the stress riser effect. Knee. 2006;13:241-3.

8. Johnson BA, Fallat LM. The effect of screw holes on bone strength. J Foot Ankle Surg. 1997;36:446-51.

9. Burstein AH, Currey J, Frankel VH, Heiple KG, Lunseth P, Vessely JC. Bone strength: the effect of screw holes. J Bone Joint Surg Am. 1972;54:1143-56.

10. Rosson J, Egan J, Shearer J, Monro P. Bone weakness after the removal of plates and screws. Cortical atrophy or screw holes? J Bone Joint Surg Br. 1991;73:283-6. 